\title{
The Era of Device Colonizers: Chryseobacterium indologenes Infections from a Tertiary Care Center in North India
}

\author{
Vidhi Jain, Chinmoy Sahu, Nayani Amrin Fatema Afzal Hussain, Malay Ghar, Kashi Nath Prasad \\ Department of Microbiology, Sanjay Gandhi Postgraduate Institute of Medical Sciences, Lucknow, Uttar Pradesh, India
}

\section{Abstract}

Background: Chryseobacterium indologenes is a hospital environment contaminant and can cause healthcare-associated infections. Methods: Patients with $C$. indologenes infections in a tertiary care center in North India for 6 months were evaluated for susceptibility patterns, comorbidities, mechanical devices, risk factors, and treatment outcomes. The organism was provisionally identified phenotypically, and identification was confirmed by the BD Phoenix automated microbiology system. Minimum inhibitory concentration values of antibiotic susceptibility were determined. Results: A total of 12 isolates of $C$. indologenes were recovered from 11 patients. Five patients had $C$. indologenes bloodstream infection (BSI), one had ventilator-associated pneumonia (VAP), and one had both BSI and VAP. In four others, the organism was isolated from the catheterized urinary tract. All VAP and BSI patients were admitted to the Intensive Care Units and mechanically ventilated; all had central lines and history of colistin therapy during the past 15 days. The common underlying risk factors were diabetes, hypertension, and coronary artery disease. Conclusions: $C$. indologenes infections are increasing because of higher use of carbapenems and colistin, to which it is intrinsically resistant.

Keywords: Antibiotics, Chryseobacterium indologenes, Intensive Care Units, opportunistic pathogens

\section{INTRODUCTION}

Chryseobacterium indologenes is a known colonizer of inanimate objects in the hospital environment. ${ }^{[1]} C$. indologenes is not a part of human microflora, but it has been increasingly isolated from patients with bacteremia, pneumonia, and artificial shunt infections, especially in those who are admitted with long-term indwelling devices and prolonged exposure to broad-spectrum antibiotics. ${ }^{[2-6]}$ Opportunistic infections due to this organism, often arising from colonization of indwelling mechanical devices, are on the rise. ${ }^{[3]}$ It has increasingly been reported from Southeast Asia, mainly from Taiwan. ${ }^{[6]}$ However, there is a paucity of data regarding its occurrence and associated risk factors in the Indian healthcare set ups. The present study was aimed to quantify the burden of $C$. indologenes infections among the patients admitted in different disciplines.

\section{Methods}

Consecutive specimens of blood, respiratory secretions, and urine received between February 15 and August 15, 2016, in

\begin{tabular}{|l|l|}
\hline \multicolumn{3}{c|}{ Access this article online } \\
\hline Quick Response Code: & Website: \\
& www.ijccm.org \\
\hline
\end{tabular}

the bacteriology laboratory of our hospital, were evaluated for the presence of Chryseobacterium spp. on routine culture. Blood specimens were cultured in BACTEC plus aerobic and anaerobic bottles (BD Diagnostics, Sparks, MD) followed by subculture on blood agar and MacConkey agar plates. Respiratory specimens (endotracheal aspirate, sputum, bronchial washing, and bronchoalveolar lavage fluid) were cultured onto blood, MacConkey, and hemin agar plates, incubated overnight at $37^{\circ} \mathrm{C}$. Urine specimens were cultured on blood agar and CHROMagar (HiChrome, HiMedia, Mumbai, India) plates using semiquantitative calibrated loop technique.

Provisional identification of Chryseobacterium spp was made if yellow-colored, oxidase-positive colony of Gram-negative bacilli with unusual susceptibility pattern of resistance to

Address for correspondence: Dr. Chinmoy Sahu Department of Microbiology, Sanjay Gandhi Postgraduate Institute of Medical Sciences, Lucknow - 226 014, Uttar Pradesh, India. E-mail: sahu.chinmoy@gmail.com

This is an open access journal, and articles are distributed under the terms of the Creative Commons Attribution-NonCommercial-ShareAlike 4.0 License, which allows others to remix, tweak, and build upon the work non-commercially, as long as appropriate credit is given and the new creations are licensed under the identical terms.

For reprints contact: reprints@medknow.com

How to cite this article: Jain V, Sahu C, Afzal Hussain NA, Ghar M, Prasad KN. The era of device colonizers: Chryseobacterium indologenes infections from a tertiary care center in North India. Indian J Crit Care Med 2018;22:537-40. 
carbapenems and colistin but susceptibility to vancomycin was observed.

Such isolates were further subjected to an automated method for identification and antibiotic susceptibility testing by BD Phoenix ${ }^{\mathrm{TM}}$ automated system (BD Diagnostic Systems, Sparks, MD). Results of antibiotic susceptibility testing were interpreted as per the Clinical and Laboratory Standards Institute guidelines 2016.

\section{RESULTS}

A total of 12 isolates (six from blood, two from endotracheal aspirates and four from urine) met the criteria of provisional identification of Chryseobacterium spp. All these isolates were confirmed as $C$. indologenes by BD Phoenix ${ }^{\mathrm{TM}}$ automated system. These isolates were recovered from 11 patients; in one patient, $C$. indologenes was isolated from blood and endotracheal aspirate who had both septicemia and ventilation-associated pneumonia (VAP).

The age of patients ranged from 17 months to 80 years with mean age of $54.23 \pm 25.39$ years. The antibiotic susceptibility profile of the isolates showed that $91.67 \%$ were sensitive to trimethoprim-sulfamethoxazole, followed by $75 \%$ to levofloxacin, $41.67 \%$ to ciprofloxacin, and $16.67 \%$ to piperacillin + tazobactam. All the isolates were resistant to cefotaxime, ceftazidime, cefepime, aztreonam, gentamicin, amikacin, chloramphenicol, imipenem, meropenem, and colistin [Table 1].

The comorbidities associated with individual patients such as diabetes, hypertension, history of stroke, coronary artery disease, end-stage renal disease, recent surgery (within the last 30 days), and history of VAP during the course of current admission are shown in Table 2. All patients had at least one mechanical device inserted (central venous catheter, ventilator, or urinary catheter) at the time of diagnosis of $C$. indologenes infection. The average time duration between hospital admission and diagnosis of $C$. indologenes infection was $15.27 \pm 12.18$ days, and the mortality was $25 \%$ [Table 2].

Antibiotic use during the current admission showed that almost all patients were prescribed at least one of fluoroquinolones, third-generation cephalosporins, carbapenems, and colistin. Concurrent steroid use was in $83.33 \%$ of cases [Table 3].

\section{Discussion}

The provisional identification of $C$. indologenes isolates is based on their yellow pigmentation and positive potassium hydroxide test, coupled with an unusual antimicrobial pattern, i.e., resistance to carbapenems and colistin, while being susceptible to vancomycin.

The isolation of an uncommon organism from clinical sample naturally sparks a debate about the clinical significance of the isolate. All the isolates of $C$. indologenes from bloodstream infection (BSI) were from febrile patients and were considered true pathogens. ${ }^{[7]}$ Both isolates of $C$. indologenes from endotracheal aspirates of patients on mechanical ventilation were considered to cause VAP as determined by clinical assessment.

The two $C$. indologenes isolates were cultured from urine obtained from percutaneous nephrostomy catheters of two

\begin{tabular}{|c|c|c|c|c|c|c|c|c|}
\hline Antibiotics & Cefotaxime & Cefepime & $\begin{array}{c}\text { Piperacillin + } \\
\text { tazobactam }\end{array}$ & Meropenem & Amikacin & $\begin{array}{l}\text { Trimethoprim + } \\
\text { sulfamethoxazole }\end{array}$ & Ciprofloxacin & Levofloxacin \\
\hline $\begin{array}{l}\text { Susceptible MIC } \\
\text { breakpoint }(\mu \mathrm{g} / \mathrm{ml})\end{array}$ & $\leq 8$ & $\leq 8$ & $\leq 16 / 4$ & $\leq 4$ & $\leq 16$ & $\leq 2 / 38$ & $\leq 1$ & $\leq 2$ \\
\hline \multicolumn{9}{|l|}{ Blood } \\
\hline Isolate $1^{*}$ & $>32$ & $>16$ & $16 / 8$ & $>8$ & $>32$ & $\leq 0.5 / 9.5$ & $>2$ & $\leq 0.5$ \\
\hline Isolate 2 & $>32$ & $>16$ & $64 / 4$ & $>8$ & $>32$ & $\leq 0.5 / 9.5$ & $\leq 0.5$ & $\leq 1$ \\
\hline Isolate 3 & $>32$ & $>16$ & $64 / 4$ & $>8$ & $>32$ & $>2 / 38$ & $>2$ & $\leq 1$ \\
\hline Isolate 4 & $>32$ & $>16$ & $>64 / 4$ & $>8$ & $>32$ & $<0.5 / 9.5$ & $\leq 0.5$ & $\leq 1$ \\
\hline Isolate 5 & $>32$ & $>16$ & $<4 / 4$ & $>8$ & $>32$ & $<0.5 / 9.5$ & $>2$ & $\leq 1$ \\
\hline Isolate 6 & $>32$ & $>16$ & $<4 / 4$ & $>8$ & $>32$ & $\leq 0.5 / 9.5$ & $\leq 0.5$ & $\leq 1$ \\
\hline \multicolumn{9}{|l|}{ ET aspirate } \\
\hline Isolate $1^{*}$ & $>32$ & $>16$ & $16 / 8$ & $>8$ & $>32$ & $\leq 0.5 / 9.5$ & $>2$ & $\leq 0.5$ \\
\hline Isolate 2 & $>32$ & $>16$ & $64 / 4$ & $>8$ & $>32$ & $\leq 0.5 / 9.5$ & $\leq 0.5$ & $\leq 1$ \\
\hline \multicolumn{9}{|l|}{ Urine } \\
\hline Isolate 1 & $>32$ & $>16$ & $>64 / 4$ & $>8$ & $>32$ & $\leq 0.5 / 9.5$ & $>2$ & $>4$ \\
\hline Isolate 2 & $>32$ & $>16$ & $>64 / 4$ & $>8$ & $>32$ & $1 / 19$ & $>2$ & $>4$ \\
\hline Isolate 3 & $>32$ & $>16$ & $>64 / 4$ & $>8$ & $>32$ & $1 / 19$ & $>2$ & $>4$ \\
\hline Isolate 4 & $>32$ & $>16$ & $>64 / 4$ & $>8$ & $>32$ & $\leq 0.5 / 9.5$ & $\leq 0.5$ & $\leq 1$ \\
\hline $\begin{array}{l}\text { Overall } \\
\text { sensitivity (\%) }\end{array}$ & 0 & 0 & 16.67 & 0 & 0 & 91.67 & 41.67 & 75 \\
\hline
\end{tabular}


Table 2: Summary of comorbidities, risk factors, mechanical device use, and outcome of patients with Chryseobacterium indologenes infections of different sites

\begin{tabular}{|c|c|c|c|c|}
\hline Parameter & $\begin{array}{c}\text { Blood }(n=6), \\
n(\%)\end{array}$ & $\begin{array}{l}\text { ET aspirate } \\
(n=2), n(\%)\end{array}$ & $\begin{array}{c}\text { Urine }(n=4) \text {, } \\
n(\%)\end{array}$ & $\begin{array}{c}\text { Total }(n=12), \\
n(\%)\end{array}$ \\
\hline Diabetes & $4(66.67)$ & $2(100)$ & $1(25)$ & $7(58.33)$ \\
\hline Hypertension & $4(66.67)$ & $2(100)$ & $3(75)$ & $9(75)$ \\
\hline Stroke & $2(33.33)$ & $2(100)$ & 0 & $4(33.33)$ \\
\hline CAD & $3(50)$ & $2(100)$ & 0 & $5(41.67)$ \\
\hline ESRD & $3(50)$ & 0 & 0 & $3(25)$ \\
\hline Malignancy & 0 & 0 & 0 & 0 \\
\hline Recent surgery & $4(66.67)$ & $2(100)$ & $3(75)$ & $9(75)$ \\
\hline Tracheostomy & $3(50)$ & $1(50)$ & 0 & $4(33.33)$ \\
\hline Ventilator use in current admission & $6(100)$ & $2(100)$ & 0 & $8(66.67)$ \\
\hline History of VAP in current admission & $6(100)$ & $2(100)$ & 0 & $8(66.67)$ \\
\hline Central line catheter & $6(100)$ & $2(100)$ & 0 & $8(66.67)$ \\
\hline Urinary catheter & $6(100)$ & $2(100)$ & $4(100)$ & $12(100)$ \\
\hline 14-day mortality & $3(50)$ & $1(50)$ & 0 & $4(33.33)$ \\
\hline In-hospital mortality & $3(50)$ & $1(50)$ & 0 & $4(33.33)$ \\
\hline $\begin{array}{l}\text { Average time between admission and diagnosis } \\
\text { of Chryseobacterium indologenes infection }\end{array}$ & $16.33 \pm 7.7$ days & $31 \pm 14.0$ days & $6.25 \pm 3.96$ days & $15.27 \pm 12.18$ days \\
\hline Average total duration of hospital stay & $39.67 \pm 10.2$ days & $48.5 \pm 6.5$ days & $16 \pm 10.12$ days & $32.45 \pm 16.37$ days \\
\hline
\end{tabular}

CAD: Coronary artery disease; ESRD: End-stage renal disease; VAP: Ventilation-associated pneumonia; ET: Endotracheal

Table 3: Exposure to drugs during 7 days prior to isolation of Chryseobacterium indologenes

\begin{tabular}{lcccc}
\hline Drug(s) & Blood $(\boldsymbol{n}=\mathbf{6}), \boldsymbol{n}(\mathbf{\%})$ & $\mathrm{ET}(\boldsymbol{n}=\mathbf{2}), \boldsymbol{n}(\mathbf{\%})$ & Urine $(\boldsymbol{n}=\mathbf{4}), \boldsymbol{n}(\mathbf{\%})$ & Total $(\boldsymbol{n}=\mathbf{1 2}), \boldsymbol{n}(\mathbf{\%})$ \\
\hline Cephalosporin alone & 0 & 0 & $3(75)$ & $3(25)$ \\
Carbapenem alone & 0 & 0 & $1(25)$ & $1(8.33)$ \\
Cephalosporin + colistin & $1(16.67)$ & 0 & 0 & $1(8.33)$ \\
Cephalosporin + carbapenem + colistin & $1(16.67)$ & $1(50)$ & 0 & $2(16.67)$ \\
Cephalosporin + carbapenem + FQ + colistin & $1(16.67)$ & $1(50)$ & 0 & $2(16.67)$ \\
Cephalosporin + carbapenem + FQ + colistin + tigecycline & $3(50)$ & 0 & 0 & $3(25)$ \\
\hline
\end{tabular}

FQ: Fluoroquinolones

patients on three consecutive days. Both patients presented with fever and were treated with levofloxacin without catheter removal. Both patients responded clinically within $48 \mathrm{~h}$, and their subsequent urine cultures were sterile. Such a presentation indicates a true infection of the percutaneous nephrostomy site, as reported previously from India. ${ }^{[8]}$

Most cases were admitted to postoperative wards (three urology, two pulmonary, two surgical gastroenterology, and two neurosurgery) followed by two cases from critical care units and one from the nephrology Intensive Care Unit. The average number of days between patient admission and the first detection of $C$. indologenes was around 20 days; hence, all were categorized under nosocomial infections/colonizations. All the 11 patients had at least one mechanical device (central line, ventilator, and Foley's catheter), suggesting the acquisition of $C$. indologenes through such mechanical devices.

The SENTRY study is the only study to have estimated the epidemiology and antimicrobial susceptibility profile of C. indologenes infections worldwide. ${ }^{[1]}$ It analyzed the profile of 50 Chryseobacterium isolates recovered from 119 sentinel sites covering the Americas, Europe, and the Asia-Pacific region from 1997 to 2001 . The study documents 12 cases from a single center in India, over a mere 6-month period. The SENTRY study found C. meningosepticum (now Elizabethkingia meningosepticum) to outnumber $C$. indologenes as the most common species isolated ( 24 vs. 20 strains), whereas C. indologenes was the only species identified in our study.

The isolates in SENTRY were more susceptible to levofloxacin (98\%) than to trimethoprim + sulphamethoxazole $(87.8 \%)$ and ciprofloxacin $(80 \%)$. The isolates from Asia-Pacific region were relatively more resistant to piperacillin-tazobactam than those from North America (50\% vs. 100\%), while susceptible toward levofloxacin was comparable $(100 \%$ vs. $98 \%)$. Our isolates were highly susceptible to trimethoprim + sulfamethoxazole $(91.67 \%)$, followed by levofloxacin (75\%). Only $16.67 \%$ and $41.67 \%$ isolates in the present study were susceptibility to piperacillin-tazobactam and ciprofloxacin compared to $80 \%$ and $80 \%$, respectively, in the SENTRY study. These findings suggest that the Indian isolates of $C$. indologenes differ from the rest of the world, and the antimicrobial susceptibility pattern obtained from the rest of the Asia-Pacific region cannot be generalized to India. 
Taiwan is the country from which maximum strains of Chryseobacterium have been reported in the world. Despite its geographical proximity to India, there is a remarkable difference in the characterization of clinical isolates. Chen et al. in their study reported a staggering 215 Chryseobacterium isolates over a 71/2-year study from a single center. ${ }^{[6]}$ In that study, the isolation rate of Chryseobacterium from patients with pneumonia was much higher than from BSIs (91 vs. 22 cases).

The risk factor profile of individual cases had some similarities. Both studies found hypertension, diabetes, stroke, and ventilator use to be the most common risk factors. The high rate of malignancy and chronic kidney disease as underlying factors reported by Chen et al. was negligible among our patient population. ${ }^{[6]}$ A significant observation was that three-fourths of our patients developed $C$. indologenes infection following surgical interventions. This might be an important risk factor among our patients, which should be explored in future studies.

There has been some debate over the need to remove indwelling mechanical devices during therapy. In most of our cases, the device was not removed, and therapy was started promptly, and the recovery rate was $75 \%$. Other studies have also reported similar successful outcome without catheter removal. ${ }^{[2,5]}$

To conclude, $C$. indologenes should be recognized as an emerging nosocomial pathogen, especially among patients admitted to postoperative and critical care units. Its association with indwelling catheters and devices is well established, but devices need not be removed while administering appropriate therapy. Our cases have additionally shown recent surgical intervention to be a possible risk factor. The organism has a peculiar susceptibility to trimethoprim + sulfamethoxazole and levofloxacin while being resistant to other commonly used antimicrobial agents for Gram-negative infections, such as carbapenems and colistin. Microbiology laboratories should provisionally identify and perform susceptibility testing for this organism to help clinicians decide effective therapeutic options.

\section{Financial support and sponsorship}

Nil.

\section{Conflicts of interest}

There are no conflicts of interest.

\section{References}

1. Kirby JT, Sader HS, Walsh TR, Jones RN. Antimicrobial susceptibility and epidemiology of a worldwide collection of Chryseobacterium spp: Report from the SENTRY antimicrobial surveillance program (1997-2001). J Clin Microbiol 2004;42:445-8.

2. Hsueh PR, Hsiue TR, Wu JJ, Teng LJ, Ho SW, Hsieh WC, et al. Flavobacterium indologenes bacteremia: Clinical and microbiological characteristics. Clin Infect Dis 1996;23:550-5.

3. Stamm WE, Colella JJ, Anderson RL, Dixon RE. Indwelling arterial catheters as a source of nosocomial bacteremia. An outbreak caused by Flavobacterium species. N Engl J Med 1975;292:1099-102.

4. Sudharani V, Asiya, Saxena NK. Chryseobacterium indologenes bacteraemia in a preterm baby. Indian J Med Microbiol 2011;29:196-8.

5. Lin YT, Jeng YY, Lin ML, Yu KW, Wang FD, Liu CY, et al. Clinical and microbiological characteristics of Chryseobacterium indologenes bacteremia. J Microbiol Immunol Infect 2010;43:498-505.

6. Chen FL, Wang GC, Teng SO, Ou TY, Yu FL, Lee WS, et al. Clinical and epidemiological features of Chryseobacterium indologenes infections: Analysis of 215 cases. J Microbiol Immunol Infect 2013;46:425-32.

7. Chou DW, Wu SL, Lee CT, Tai FT, Yu WL. Clinical characteristics, antimicrobial susceptibilities, and outcomes of patients with Chryseobacterium indologenes bacteremia in an Intensive Care Unit. Jpn J Infect Dis 2011;64:520-4.

8. Garg S, Appannanavar SB, Mohan B, Taneja N. Pyonephrosis due to Chryseobacterium Gleum: A first case report. Indian J Med Microbiol 2015;33:311-3. 